\title{
Acquisition of epithelial-mesenchymal transition is associated with Skp2 expression in paclitaxel-resistant breast cancer cells
}

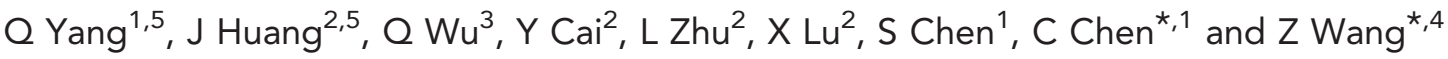 \\ ${ }^{1}$ Department of Biochemistry and Molecular Biology, Bengbu Medical College, Anhui 233030, China; ${ }^{2}$ Clinical Testing and \\ Diagnose Experimental Center of Bengbu Medical College, Anhui 233000, China; ${ }^{3}$ Department of Medical Oncology, \\ First Affiliated Hospital of Bengbu Medical College, Bengbu, Anhui 233004, China and ${ }^{4}$ Cyrus Tang Hematology Center, Jiangsu \\ Institute of Hematology, First Affiliated Hospital of Soochow University, Suzhou, Jiangsu 215123, China
}

Background: Breast cancer is the most common female malignant disease, and the second leading cause of cancer-related death in the United States. Acquired resistance to chemotherapeutic drugs is a pivotal reason that leads to worse treatment outcome of breast cancer. Therefore, it is urgent to elucidate the mechanism of drug resistance in breast cancer.

Methods: To investigate the underlying molecular basis of the acquired resistant cells to paclitaxel in breast cancer, we used multiple methods including real-time RT-PCR, western blotting analysis, migration and invasion assays, wound healing assay, and transfection.

Results: We found that epithelial-mesenchymal transition (EMT) is involved in paclitaxel-resistant (PR) breast cancer cells. The resistant cells with EMT features exhibit increased migration and invasion activities. Mechanistically, high expression of Skp2 was found to be associated with EMT in PR cells. Notably, depletion of Skp2 in PR cells led to partial reversal of EMT phenotype.

Conclusions: These findings suggest that Skp2 was critically involved in PR-mediated EMT. Skp2 could be a potential therapeutic target for breast cancer.

Breast cancer is one of the most common malignancies among women, and the second leading cause of cancer-related mortality in the United States (Siegel et al, 2013). It is estimated that 232340 new cases and 39620 deaths will be occurred in 2013 (Siegel et al, 2013). Current therapies for breast cancer in clinic include surgery, chemotherapy, radiation, and hormonal therapies (ChavezMacGregor and Gonzalez-Angulo, 2013). Among these therapies, chemotherapy is one of the major treatments for breast cancer (Zardavas et al, 2013). However, intrinsic or acquired resistance to chemotherapeutic drug leads to failure of chemotherapy in many patients with breast cancer (Ali and Coombes, 2002; Holohan et al, 2013). Therefore, it is critical to explore the molecular mechanisms of drug resistance in breast cancer, and find novel therapeutic targets for overcoming drug resistance and improving the survival of breast cancer patients.
Paclitaxel, also known as taxol, as one of the most effective chemotherapeutic drugs has been used to treat human cancers including breast cancer (Hernandez-Aya and Gonzalez-Angulo, 2013). Unfortunately, many patients developed drug resistance during paclitaxel treatment. Multiple studies have demonstrated that epithelial-mesenchymal transition (EMT) is involved in acquired resistance to paclitaxel in human malignancies (Kajiyama et al, 2007; Du et al, 2013). Epithelial-mesenchymal transition is a complex process by which epithelial cells transit to mesenchymal phenotype through loss of epithelial cell-cell junction and actin cytoskeleton reorganisation (De Craene and Berx, 2013). After EMT, cells lose the expression of epithelial marker E-cadherin, but gain the expression of mesenchymal markers such as Vimentin, Snail, Slug, ZEB1 (zinc-finger E-box binding homeobox 1), and ZEB2 (Thiery et al, 2009).

\footnotetext{
*Correspondence: Dr Professor C Chen; E-mail: tochenchangjie@163.com or Dr Professor Z Wang; E-mail: zhiwei@gmail.com
} ${ }^{5}$ These authors contributed equally to this work. 
It has been reported that chemoresistance to paclitaxel induced EMT and enhanced metastatic potential in ovarian carcinoma cells (Kajiyama et al, 2007). Moreover, paclitaxel-resistant (PR) ovarian cancer cells acquired EMT in part due to overexpression of phosphatidylinositol 3-kinase (PI3K) (Du et al, 2013). Emerging evidence also showed that PR breast cancer cells have higher expression of P-glycoprotein, lung resistance-related protein, and glutathione-S-transferase- $\pi$, which could be involved in paclitaxel resistance (Chen et al, 2013). Although these studies revealed the paclitaxel resistance mechanism, the molecular basis for paclitaxel resistance has not been fully elucidated.

Skp2 (S-phase kinase-associated protein 2) has been found to have critical roles in the breast tumorigenesis (Wang et al, 2012a). Skp2 exerts its oncogenic functions through degradation of its ubiquitination targets such as p21 (Yu et al, 1998), p27 (Tsvetkov et al, 1999), p57 (Kamura et al, 2003), E-cadherin (Inuzuka et al, 2012), and FOXO1 (Huang et al, 2005). Emerging evidence has revealed that Skp2 has important roles in cell growth, apoptotic cell death, migration, invasion, and metastasis in human breast cancer (Zheng et al, 2005; Sonoda et al, 2006). Overexpression of Skp2 has been observed in breast cancer cell lines and primary breast tumours (Radke et al, 2005; Fujita et al, 2008). Moreover, high expression of Skp2 is correlated with tumour recurrence in human cancers (Einama et al, 2006). Furthermore, the positive relationship between Skp2 expression and tumour metastasis has been reported in human malignancies (Tosco et al, 2011). Notably, Skp2 overexpression was detected more frequently in tumours metastatic to the lymph nodes in breast cancer (Zheng et al, 2005). Consistently, depletion of Skp2 restricted breast cancer metastasis to the lung, whereas overexpression of Skp2 promoted the metastatic events (Chan et al, 2010). Several studies have shown that Skp2 is involved in drug resistance in human cancers including breast cancer (Ishii et al, 2004; Davidovich et al, 2008). Recently, one study showed that TGF- $\beta 1$ induced EMT partly through upregulation of Skp2 in melanomas, indicating that Skp2 could have a role in EMT progress (Qu et al, 2014). As Skp2 is involved in drug resistance and EMT, it is urgent to investigate whether Skp2 is critical involved in drug resistant-mediated EMT in breast cancer. In the current study, we established PR breast cancer cell lines. We further investigate whether these cells acquired EMT phenotype and features, and whether Skp2 has a pivotal role in PR-mediated EMT.

\section{MATERIALS AND METHODS}

Cell culture, reagents and antibodies. Human breast cancer cell lines MCF-7 and SKBR3 were cultured in RPMI 1600 (Invitrogen, Carlsbad, CA, USA) supplemented with $10 \%$ fetal bovine serum (FBS), penicillin, and streptomycin, and maintained in a humidified $5 \% \mathrm{CO}_{2}$ incubator at $37^{\circ} \mathrm{C}$. MTT [3-(4,5-dimethythiazol- 2-yl)-2,5-diphenyl tetrazolium bromide] was purchased from Sigma (St Louis, MO, USA). Primary antibodies against Vimentin, Snail, Slug, ZEB1, p21, p27, p57, and $\beta$-actin were bought from Santa Cruz Biotechnology (Santa Cruz, CA, USA). Anti-Skp2 and anti-FOXO1 antibodies were purchased from Abcam (Cambridge, MA, USA). MCF-7 and SKBR3 cells were exposed to increasing concentrations of paclitaxel for more than 6 months to create PR cell lines.

MTT assay. The cells $\left(5 \times 10^{3}\right)$ were seeded in each well of the 96-well plates for overnight incubation. Then, the cells were treated with different concentrations of paclitaxel for $72 \mathrm{~h}$. MTT assay was performed as described previously (Wu et al, 2013).

Cell attachment and detachment assay. Cell attachment and detachment assays were performed as described before (Kong et al, 2008). Briefly, for attachment assay, $5 \times 10^{4}$ cells per well were seeded in 24-well plates. After $1 \mathrm{~h}$ incubation, the unattached cells were removed and attached cells were counted. For cell detachment assay, after $24 \mathrm{~h}$ incubation, the detached cells by $0.05 \%$ trypsin for $3 \mathrm{~min}$ were counted. The remaining attached cells were also counted after trypsinisation. Data were presented as a percentage of the attached or detached cells to total cells.

Transwell migration and invasion assays. Cell migration was assessed using 24 -well inserts with $8 \mu \mathrm{m}$ pores as described before (Wu et al, 2013). The invasive activity of cells was detected by using BD BioCoat Tumor Invasion Assay System (BD Biosciences, Bedford, MA, USA) following the protocol provided by the manufacturer as described earlier (Wu et al, 2013). Briefly, cells were added to the upper chamber of the inserts. RPMI 1600 medium with $10 \%$ FBS was added to the lower chamber. The cells were allowed to migrate for $12 \mathrm{~h}$ at $37^{\circ} \mathrm{C}$. After removing cells on the upper side of the transwell, the invading cells on the underside were fixed and stained with Giemsa solution. The stained invasive cells were photographed under a microscope.

Wound healing assay. The cells were seeded in six-well plate until the cells grew to $90 \%$ confluency. The scratch wound was generated by a pipette tip in the surface of the plates. Photographic images were taken at 0 and $16 \mathrm{~h}$.

Quantitative RT-PCR analysis for gene expression. The total RNA was isolated with Trizol (Invitrogen) according to the manufacturer's protocols. The relative quantitative real-time PCR reactions were described previously (Wu et al, 2013). The primers used in PCR reaction are below: Skp2, forward primer (5'-TGC TAA GCA GCT GTT CCA GA-3') and reverse primer (5'-AAG ATT CAG CTG GGT GAT GG); E-cadherin, forward primer (5'-GAA GTG TCC GAG GAC TTT GG-3') and reverse primer (5'-CAG TGT CTC TCC AAA TCC GAT A-3'); Vimentin, forward primer (5'-TGT CCA AAT CGA TGT GGA TGT TTC-3') and reverse primer $\left(5^{\prime}\right.$-TTG TAC CAT TCT TCT GCC TCC TG-3'); Snail, forward primer ( $5^{\prime}$-CGG AAG CCT AAC TAC AGC GA-3') and reverse primer ( $5^{\prime}$-GGA CAG AGT CCC AGA TGA GC-3'); Slug, forward primer ( $5^{\prime}$-CAT GCC TGT CAT ACC ACA AC- $\left.3^{\prime}\right)$ and reverse primer $\left(5^{\prime}\right.$-GGT GTC AGA TGG AGG AGG G-3'); GAPDH, forward primer $\left(5^{\prime}\right.$-CAG CCT CAA GAT CAT CAG CA-3 $\left.3^{\prime}\right)$ and reverse primer $\left(5^{\prime}\right.$-TGT GGT CAT GAG TCC TTC CA-3').

Western blotting analysis. Cells were lysed with RIPA buffer ( $50 \mathrm{~mm}$ Tris, $150 \mathrm{~mm} \mathrm{NaCl}, 1 \%$ TritonX-100, 0.1\% sodium dodecyl sulphate, and $1 \%$ nadeoxycholate) supplemented with protease inhibitors. The protein concentrations were determined using the Bio-Rad protein assay kit (Bio-Rad Laboratories, Hercules, CA, USA). The proteins were separated by SDS-PAGE and then electrotransferred to membranes. These membranes were then immunoblotted with indicated antibodies for western blotting as described previously (Tan et al, 2014).

Cell cycle analysis. Cells were plated at $1 \times 10^{5}$ cells/well on six-well plates. After $48 \mathrm{~h}$, cells were washed twice with cold PBS and fixed with $70 \%$ ethanol overnight at $4^{\circ} \mathrm{C}$. Then, the cells were resuspended to $1 \times 10^{6} \mathrm{cells} / \mathrm{ml}$ in PBS and incubated with $100 \mu \mathrm{g} \mathrm{ml}^{-1}$ RNase A and $50 \mathrm{mg} \mathrm{ml}^{-1}$ propidium iodide (PI) at room temperature for $30 \mathrm{~min}$. The distribution of the cells throughout cell cycle was determined by flow cytometry.

Transfection. Cells were seeded in six-well plates and transfected with Skp2 siRNA, or control siRNA using Lipofectamine 2000 as described before (Wu et al, 2013). The sequences used for Skp2 siRNA are as followed: Skp2 siRNA1, Forward oligo, $5^{\prime}$-GGA GUG ACA AAG ACU UUG UTT-3'; Reverse oligo, 5' -ACA AAG UCU UUG UCA CUC CTT -3'; Skp2 siRNA 2, Forward oligo, 5'-GUG AUA GUG UCA UGC UAA ATT-3'; Reverse oligo, 5' -UUU AGC AUG ACA CUA UCA CTT-3'; Skp2 siRNA 3, Forward oligo, 
5'-GUC GGU GCU AUG AUA UAA UTT-3'; Reverse oligo, $5^{\prime}$-AUU AUA UCA UAG CAC CGA CTT-3'. The non-targeting control siRNA, Forward oligo, $5^{\prime}$-UUC UCC GAA CGU GUC ACG UTT-3'; Reverse oligo, 5'-ACG UGA CAC GUU CGG AGA ATT-3'. After the transfection, the cells were used for further analysis as described under the results section.

Statistical analysis. Each experiment consisted of three replications. Statistical comparisons between different groups were evaluated using GraphPad Prism 4.0 (Graph pad Software, La Jolla, CA, USA). Statistical analyses were performed with mean \pm standard deviation (s.d.) values. $P<0.05$ was considered statistically significant.

\section{RESULTS}

Establishment of PR breast cancer cell lines. To explore the mechanism of drug resistance in breast cancer, we established the PR breast cancer cells. MCF-7 and SKBR3 cells were exposed to increasing concentrations of paclitaxel for more than 6 months. As illustrated in Figure $1 \mathrm{~A}, 10 \mu \mathrm{g} \mathrm{ml}^{-1}$ paclitaxel led to $\sim 50$ and
$70 \%$ cell growth inhibition in SKBR3 and MCF-7 cells, respectively. However, PR cells exhibited resistance to the growth inhibitory properties of $10 \mu \mathrm{g} \mathrm{ml}^{-1}$ paclitaxel (Figure 1A). The resistant cells were continuously maintained in RPMI1600 medium containing $10 \mu \mathrm{g} \mathrm{ml}^{-1}$ paclitaxel for the current study.

PR cells acquire EMT feature. Multiple studies have demonstrated that drug-resistant cells exhibited EMT phenotype (Wang et al, 2011; Mallini et al, 2014). Consistent with this notion, we observed that MCF7 PR and SKBR3 PR cells acquired the markedly morphologic changes, which were associated with EMT phenotype. Both MCF7 PR and SKBR3 PR cells exhibited elongated, fibroblastoid morphology, whereas MCF7 and SKBR3 displayed a rounded shape (Figure 1B). It has been well documented that induction of EMT was associated with aggressive characteristics, such as cell attachment, detachment, migration, and invasion. In line with this concept, we found that PR cells have significantly increased numbers of invaded cells through a Matrigel-coated membrane compared with parental cells (Figure 1C). Moreover, our results showed that PR cells have enhanced capacity of attachment and detachment compared with parental cells (Figure 1D). Furthermore, our wound healing assay
A

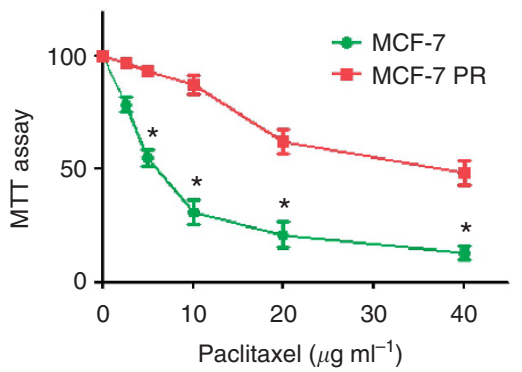

B

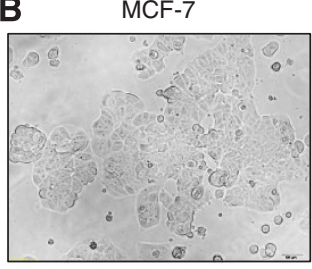

C

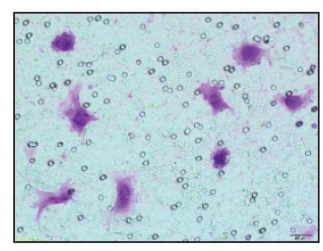

。

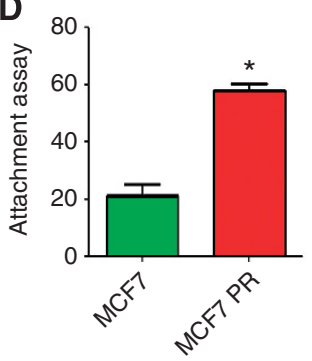

MCF-7 PR

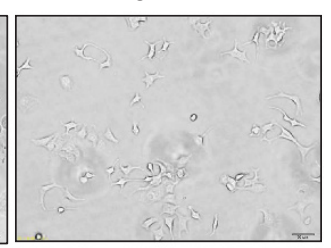

MCF-7 PR
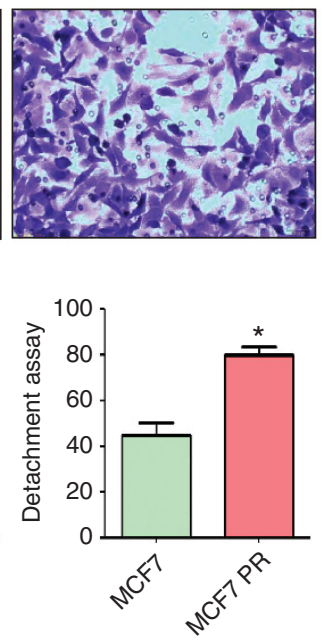
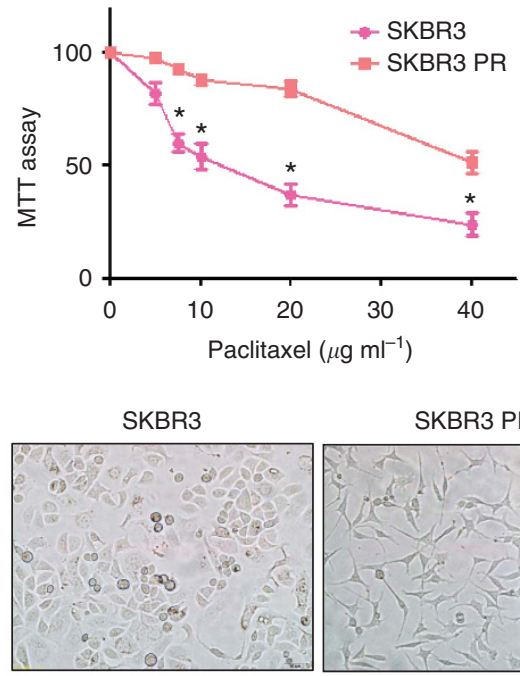

SKBR3 PR

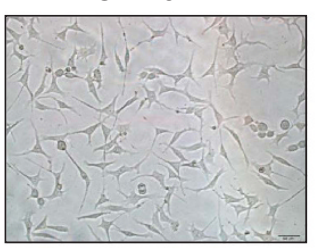

SKBR3 PR
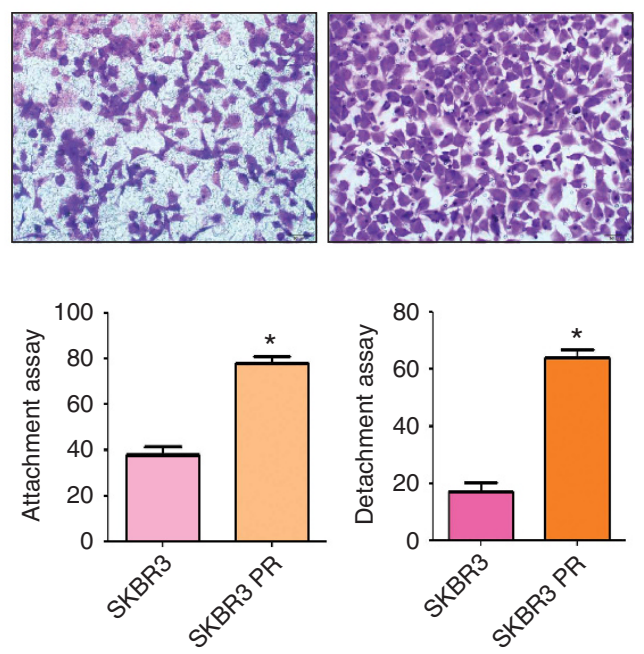

Figure 1. PR cells exhibited EMT phenotype. (A) MTT assay was conducted in parental and PR breast cancer cells. ${ }^{\star} P<0.05$ vs control. (B) Cell morphology was observed by microscopy in parental and PR cells. Parental MCF7 and SKBR3 cells displayed an epithelioid appearance, whereas their PR cells showed elongated, irregular fibroblastoid morphology. (C) Invasion assay was performed to measure the invasive capacity in parental and PR cells. (D) Cell attachment and attachment assays were assessed in parental and PR cells. ${ }^{\star} P<0.05$ vs control. 
revealed that PR cells have enhanced motility activity (Figure $2 \mathrm{~A}$ ). Taken together, these findings suggest that PR cells acquire EMT characteristics.

PR cells have EMT molecular marker changes. To validate whether PR cells have EMT molecular marker changes, we compared the expression of EMT markers in paired parental and resistant cell lines by RT-PCR and western blotting analysis, respectively. We found that epithelial molecule E-cadherin was significantly decreased in PR cells, whereas the expression of mesenchymal markers such as Snail, Vimentin, and Slug was highly elevated in PR cells (Figure $2 \mathrm{~B}$ and $\mathrm{C}$, Figure 3). These results further confirmed that PR cells acquired a mesenchymal phenotype, which could be involved in paclitaxel resistance in breast cancer.

Overexpression of Skp2 was found in PR cells. Recently, emerging evidence has demonstrated that Skp2 is involved in EMT in human cancer. To explore whether Skp2 has a critical role in PR-mediated EMT, we measured the expression of Skp2 at mRNA and protein levels in PR cells and parental cells using RT-PCR and western blotting, respectively. As expected, we found that the expression of Skp2 at mRNA and protein levels was significantly elevated in PR cells compared with parental cells (Figure 4A and B). Consistently, the expression of Skp2 substrates including p21, p27, p57, and FOXO1 were decreased in PR cells (Figure 4B). Moreover, cell cycle analysis results showed that it has an increased $S$ phase in PR cells (Figure 4C). These findings indicated that the acquisition of EMT could be in part due to overexpression of Skp2 in PR cells.

Depletion of Skp2 reverses EMT to MET in PR cells. To further investigate whether Skp2 has a critical role in PR-induced EMT, we depleted the expression of Skp2 in PR cells. As demonstrated in Figure 4D, Skp2 siRNA2 transfection significantly inhibited the expression of Skp2 in PR cells. Then, we used Skp2 siRNA2 to explore whether depletion of Skp2 could reverse EMT to mesenchymal-epithelial transition (MET) in PR cells. Indeed, we found that PR cells with Skp2 siRNA treatment exhibited round cell-like morphology (Figure 4E), suggesting that downregulation of Skp2 partly reversed EMT to MET phenotype. In support of this, depletion of Skp2 by its siRNA in PR cells inhibited cell attachment and detachment capacity (Figure 4F).

Depletion of Skp2 reduces motility and invasion in PR cells. To further validate the role of Skp2 in PR cells, we detected the cell motility and invasion capacities in PR cells after Skp2 siRNA transfection. Our results showed that depletion of Skp2 markedly inhibited the migration and invasion in PR cells (Figure 5A-C). Consistent with these results, our wound healing assay

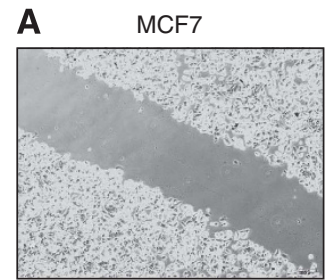

$\mathrm{Oh}$

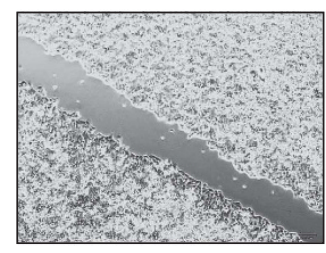

$20 \mathrm{~h}$

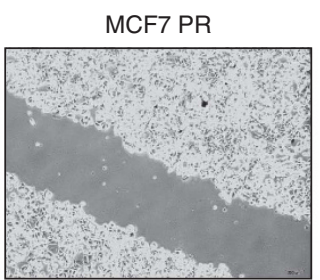

$\mathrm{Oh}$

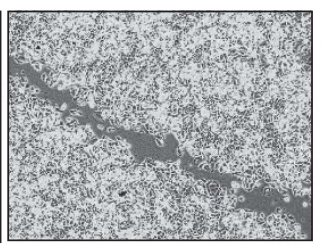

$20 \mathrm{~h}$

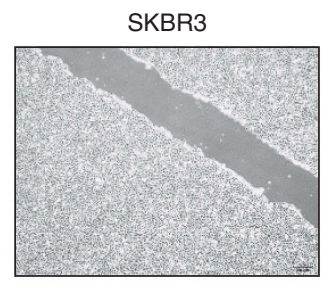

$\mathrm{Oh}$

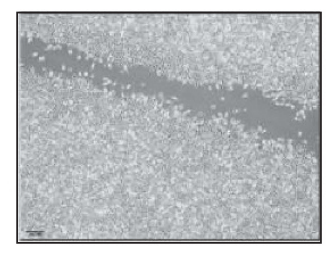

$20 \mathrm{~h}$
SKBR3 PR

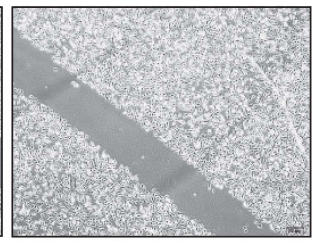

$\mathrm{Oh}$

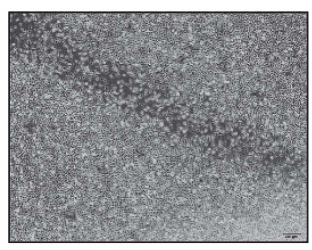

$20 \mathrm{~h}$

B
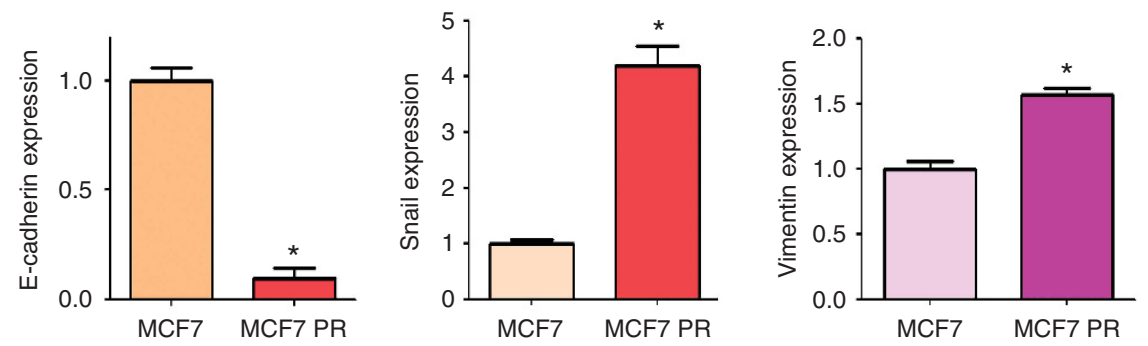

C
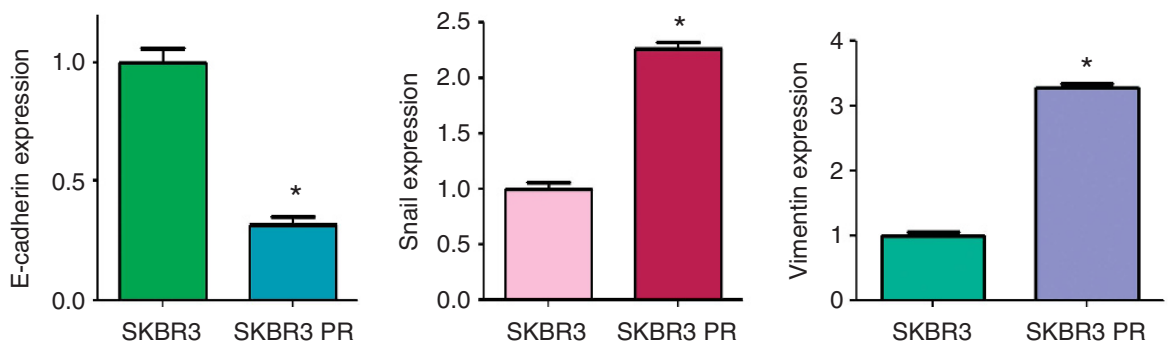

Figure 2. PR cells have enhanced motility activity. (A) Wound assays were performed in parental and PR cells. (B and C) Real-time RT-PCR assay was conducted to detect the expression of EMT markers in parental and PR cells. * $P<0.05$ PR vs control. 

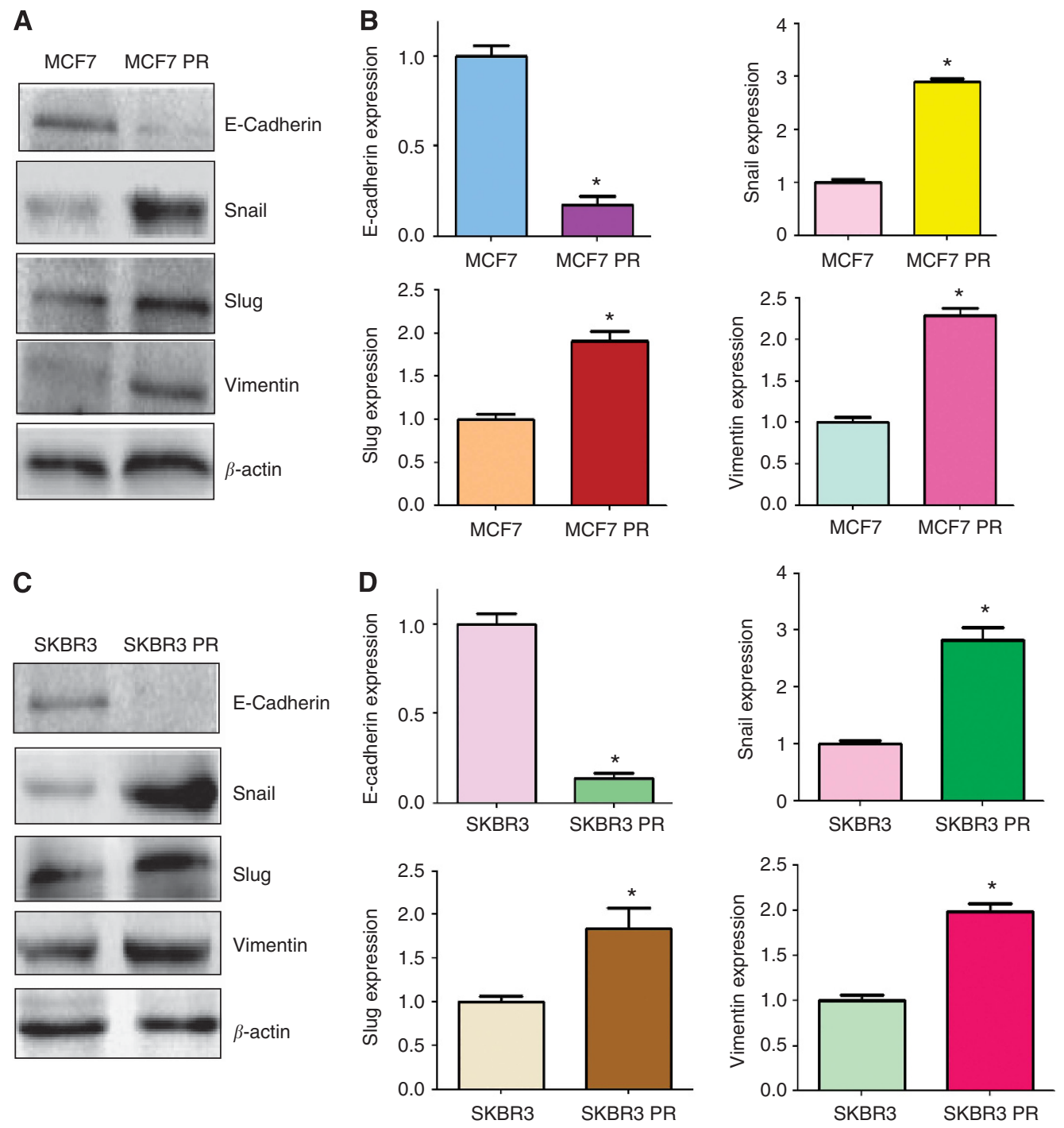

Figure 3. PR cells have EMT marker changes. (A) Western blotting analysis was used to detect the expression of E-cadherin, Snail, Slug, and Vimentin in MCF7 and MCF7 PR cells. (B) Quantitative results are illustrated for panel A. ${ }^{\star} P<0.05$ vs control. (C) Western blotting analysis was performed to measure the expression of E-cadherin, Snail, Slug, and Vimentin in SKBR3 and SKBR3 PR cells. (D) Quantitative results are illustrated for panel C. ${ }^{\star} P<0.05$ vs control.

demonstrated that Skp2 siRNA reduced cell motility in PR cells (Figure 5D). These findings revealed that Skp2 is critically involved in cell migration and invasion characteristics in PR cells.

Depletion of Skp2 regulates expression of EMT markers. To define whether depletion of Skp2 changes the expression of EMT markers in PR cells, we measured the expression of EMT molecules in PR cells transfected with Skp2 siRNA by real-time RT-PCR and western blotting analysis. We found that the expression of epithelial marker E-cadherin was highly increased in PR cells after depletion of Skp2, whereas the expression of mesenchymal markers including Vimentin, Snail, and Slug was significantly deceased in PR cells with Skp2 siRNA transfection (Figure 6). Altogether, our results identified that Skp2 is involved in regulation of EMT in PR cells.

Downregulation of Skp2 enhances PR cells to paclitaxel sensitivity. To investigate whether downregulation of Skp2 expression enhances PR cells to paclitaxel sensitivity, we performed MTT assay in Skp2 siRNA transfected PR cells. We found that depletion of Skp2 significantly attenuated cell growth inhibition around $30-35 \%$ induced by $10 \mu \mathrm{g} \mathrm{ml}^{-1}$ paclitaxel (Figure $7 \mathrm{~A}$ ). These results suggested that Skp2 siRNA-transfected PR cells were significantly more sensitive to paclitaxel-induced cell growth inhibition.

\section{DISCUSSION}

Breast cancer is one of the most common causes of death in women in the United States (Siegel et al, 2013). Chemotherapeutic drugs such as paclitaxel against breast cancer have only modestly effective due to acquired drug resistance. Therefore, it is necessary to define the mechanism of drug resistance in breast cancer. To this end, in the current study, we explored the molecular mechanism of paclitaxel resistance in breast cancer cells. We found that PR breast cancer cells acquired EMT characteristics and had increased motility and invasion activities. Moreover, overexpression of Skp2 was observed in PR cells. More importantly, depletion of Skp2 partly reversed EMT to MET, suggesting that Skp2 is involved in chemoresistance and EMT characteristics of breast cancer cells. Thus, targeting Skp2 could be a novel strategy for overcoming drug resistance and successful treatment of breast cancer. 
A

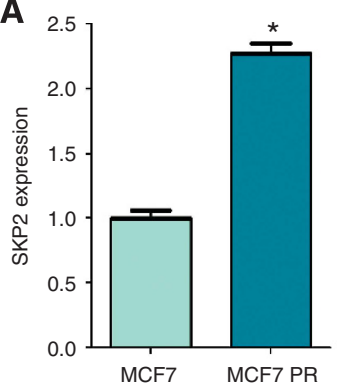

C

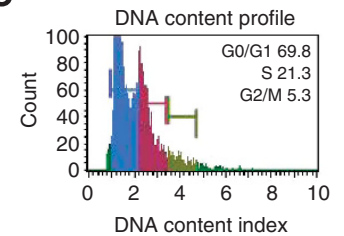

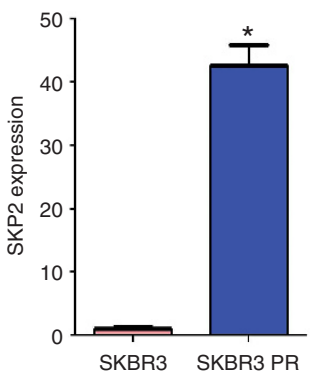

MCF7 PR

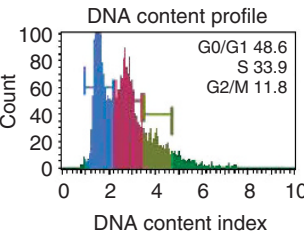

B

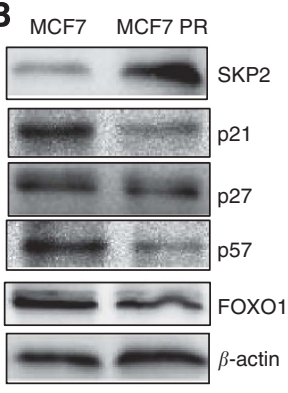

SKBR3

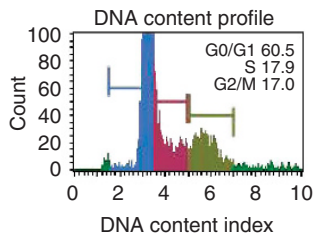

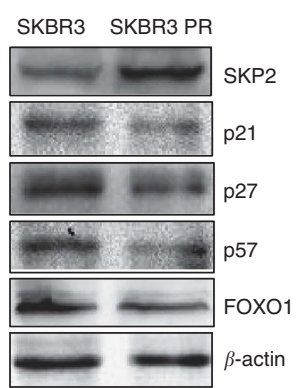

SKBR3 PR

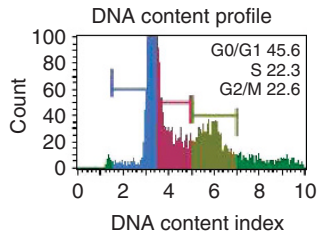

D MCF7 PR

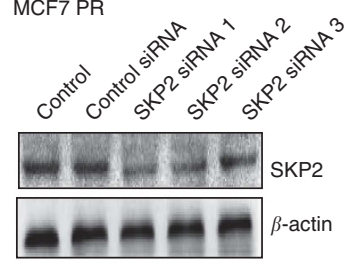

E

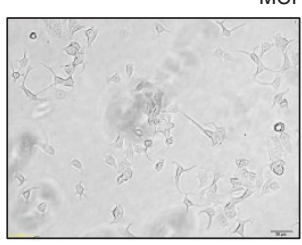

Control siRNA

F

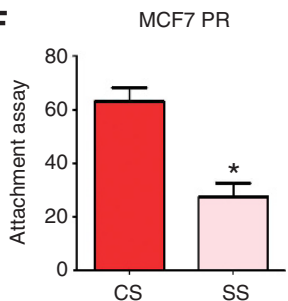

MCF7 PR

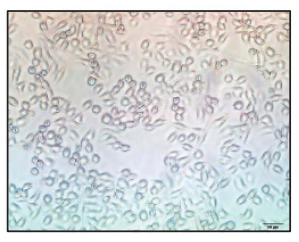

SKP2 siRNA

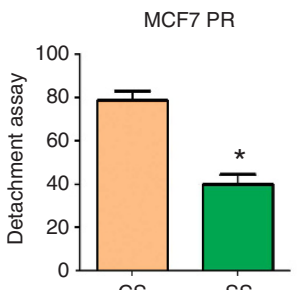

SKBR3 PR

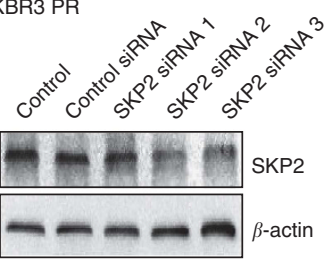

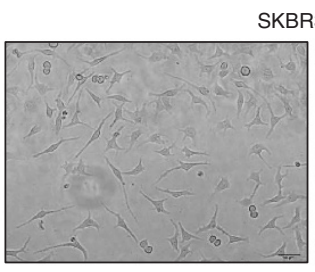

Control siRNA

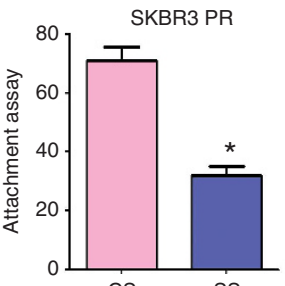

ss
SKBR3 PR

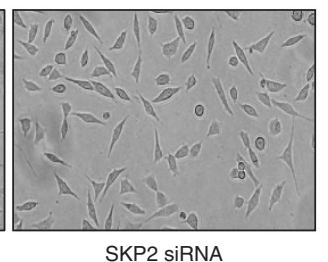

SKBR3 PR

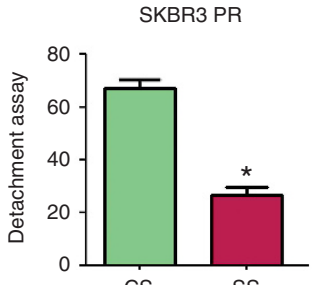

Figure 4. PR cells have high expression of Skp2. (A) Real-time RT-PCR assay was conducted to detect the expression of Skp2 in parental and PR cells. ${ }^{*} P<0.05$ PR vs control. (B) Western blotting analysis was performed to detect the expression of Skp2 and its target genes in parental and PR cells. (C) Cell cycle analysis was performed by PI staining and flow cytometry in parental and PR cells. (D) Western blotting analysis was performed to detect the efficacy of Skp2 siRNA transfection. (E) Cell morphology was taken by microscopy in PR cells transfected with Skp2 siRNA. (F) Cell attachment and detachment assays were measured in PR cells transfected with Skp2 siRNA. * $P<0.05$ vs control. Abbreviations: CS =control siRNA; SS = Skp2 siRNA.

A growing body of literature strongly suggests that there is a link between EMT and chemoresistant cancer cells. Our previous study showed that gemcitabine-resistant pancreatic cancer cells underwent EMT progression through Notch signalling pathway (Wang et al, 2009). Similarly, gemcitabine-resistant hepatocellular carcinoma cells acquired EMT characteristics with decreased E-cadherin and increased Vimentin, Snail, and Slug (Wu et al, 2013). Paclitaxel-resistant ovarian cancer cells exhibited EMT phenotype and high expression of PI3K (Kajiyama et al, 2007; Du et al, 2013). In line with these reports, our study demonstrated that PR breast cancer cells displayed EMT phenotype with downregulation of E-cadherin and upregulation of Vimentin, Snail, and Slug.
Furthermore, PR breast cancer cells have enhanced activities of migration and invasion. Our findings suggest that paclitaxel resistance is associated with EMT in breast cancer.

Skp2 has been found to be involved in drug resistance and EMT (Chan et al, 2012). For example, it has been reported that Skp2 confers resistance of pancreatic cancer cells towards the tumour necrosis factor-related apoptosis-inducing ligand-induced apoptosis (Schuler et al, 2011). Overexpression of Skp2 increased chemoresistance against camptothecin and cisplatin in human lung cancer cells (Ishii et al, 2004). In line with this, overexpression of Skp2 is associated with resistance to preoperative doxorubicinbased chemotherapy in breast cancer (Davidovich et al, 2008). 
A

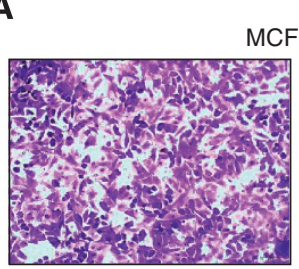

Control siRNA
MCF7 PR

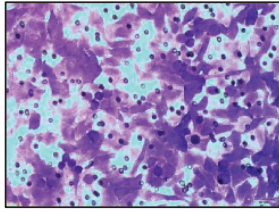

SKP2 SiRNA

Migration

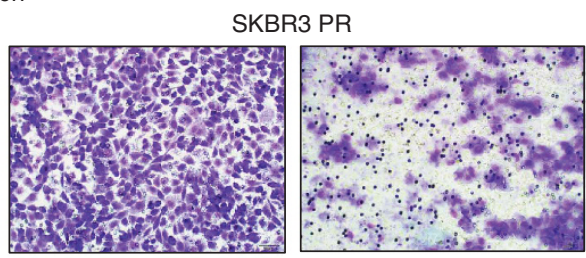

Control siRNA

SKP2 SiRNA

B

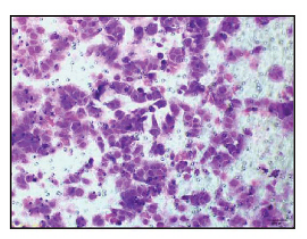

Control siRNA

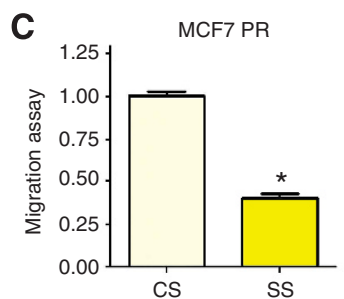

MCF7 PR

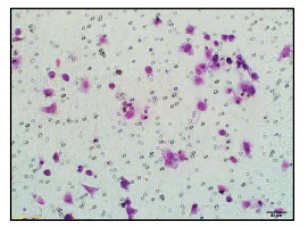

SKP2 SiRNA

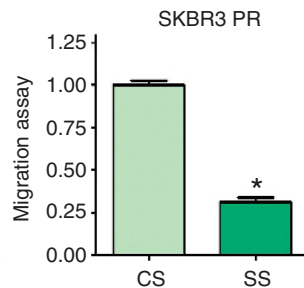

MCF7 PR

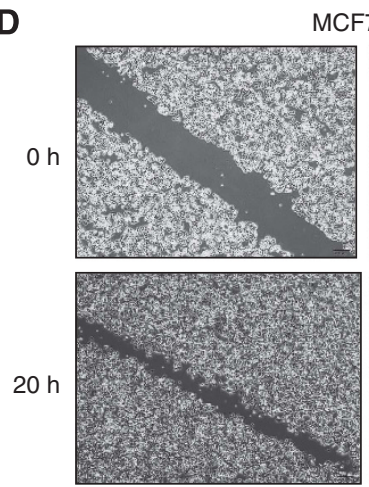

Control siRNA
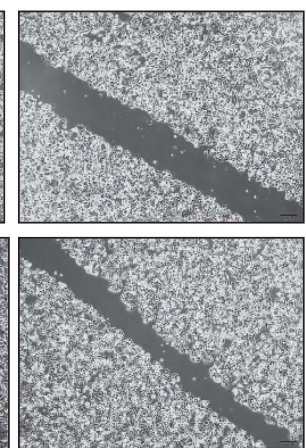

SKP2 SIRNA

Invasion

SKBR3 PR

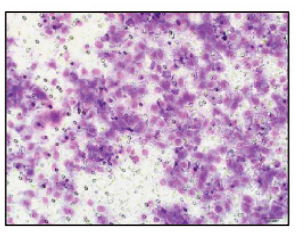

Control siRNA
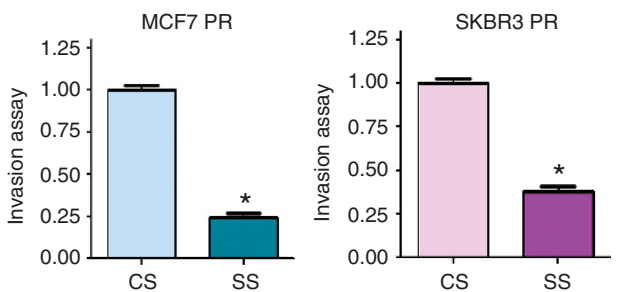

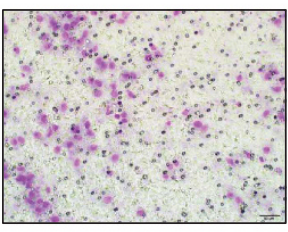

SKP2 siRNA

SKBR3 PR

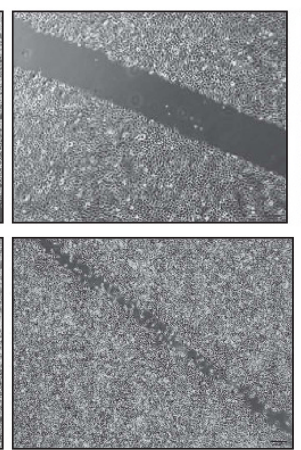

Control siRNA
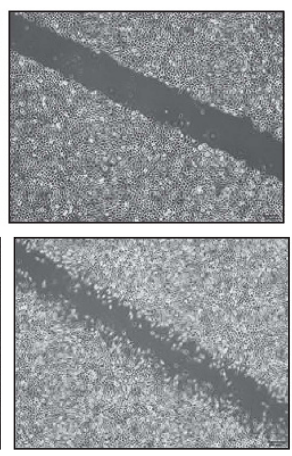

SKP2 siRNA

Figure 5. Depletion of Skp2 inhibits motility and invasion in PR cells. (A) Migration assay were conducted in PR cells transfected with Skp2 siRNA. (B) Invasion assay were performed in PR cells transfected with Skp2 siRNA. (C) Quantitative results are illustrated for panel A and B. ${ }^{\star} P<0.05$ vs control. (D) Wound healing assays were used to detect the motility in PR cells transfected with Skp2 siRNA.

Moreover, depletion of Skp2 sensitises Her2-overexpressing tumours to Herceptin treatment (Chan et al, 2012). In support the role of Skp2 in drug resistance, overexpression of Skp2 was observed in PR breast cancer cells. Recently, we found that Skp2 promoted E-cadherin ubiquitination and degradation (Inuzuka et al, 2012). Consistently, we observed an inverse correlation between Skp2 and E-cadherin expression in breast tumour samples (Inuzuka et al, 2012; Wang et al, 2012b). It has been known that E-cadherin suppression, as a hallmark of EMT, has a critical role during EMT. Several factors such as Snail, ZEB, and Twist mediated EMT through inhibition of E-cadherin expression (Gheldof and Berx, 2013). As E-cadherin is a substrate of Skp2 (Inuzuka et al, 2012), Skp2 regulated EMT partly through degradation of E-cadherin. More recently, one study has shown that TGF- $\beta 1$ induced EMT partly through upregulation of Skp2 (Qu et al, 2014). These findings indicated that Skp2 could regulate EMT in human cancer. Indeed, we observed the high expression of Skp2 in EMT-type cells. Strikingly, depletion of Skp2 caused the reversal of EMT to MET phenotype in PR cells. Altogether, our findings provide an insight into the mechanisms involved in paclitaxel resistance.

In summary, for the first time, our findings revealed that PR cells underwent EMT in part due to overexpression of Skp2 signalling pathway (Figure 7B). Furthermore, downregulation of Skp2 enhanced PR cells to paclitaxel sensitivity. These results also demonstrated that inactivation of Skp2 could be a promising strategy for restoring sensitivity to paclitaxel. Thus, Skp2 could be a potential molecular target for the treatment of breast cancers with acquired resistance to paclitaxel. To this end, several small molecule inhibitors including Compound A, SMIP0004, and Compound 25 (also known as SZL-P1-41) that block the Skp2 have been developed through a high-throughput screening (Chen et al, 2008; Rico-Bautista et al, 2010; Chan et al, 2013). Interestingly, natural agents including curcumin, quercetin, lycopene, silibinin, epigallocatechin-3-gallate, and Vitamin D3 could downregulate Skp2 expression in human cancers (Yang and Burnstein, 2003; Roy et al, 2007; Huang et al, 2008, 2011). Owing to the non-toxic nature of natural agents, inactivation of Skp2 by 

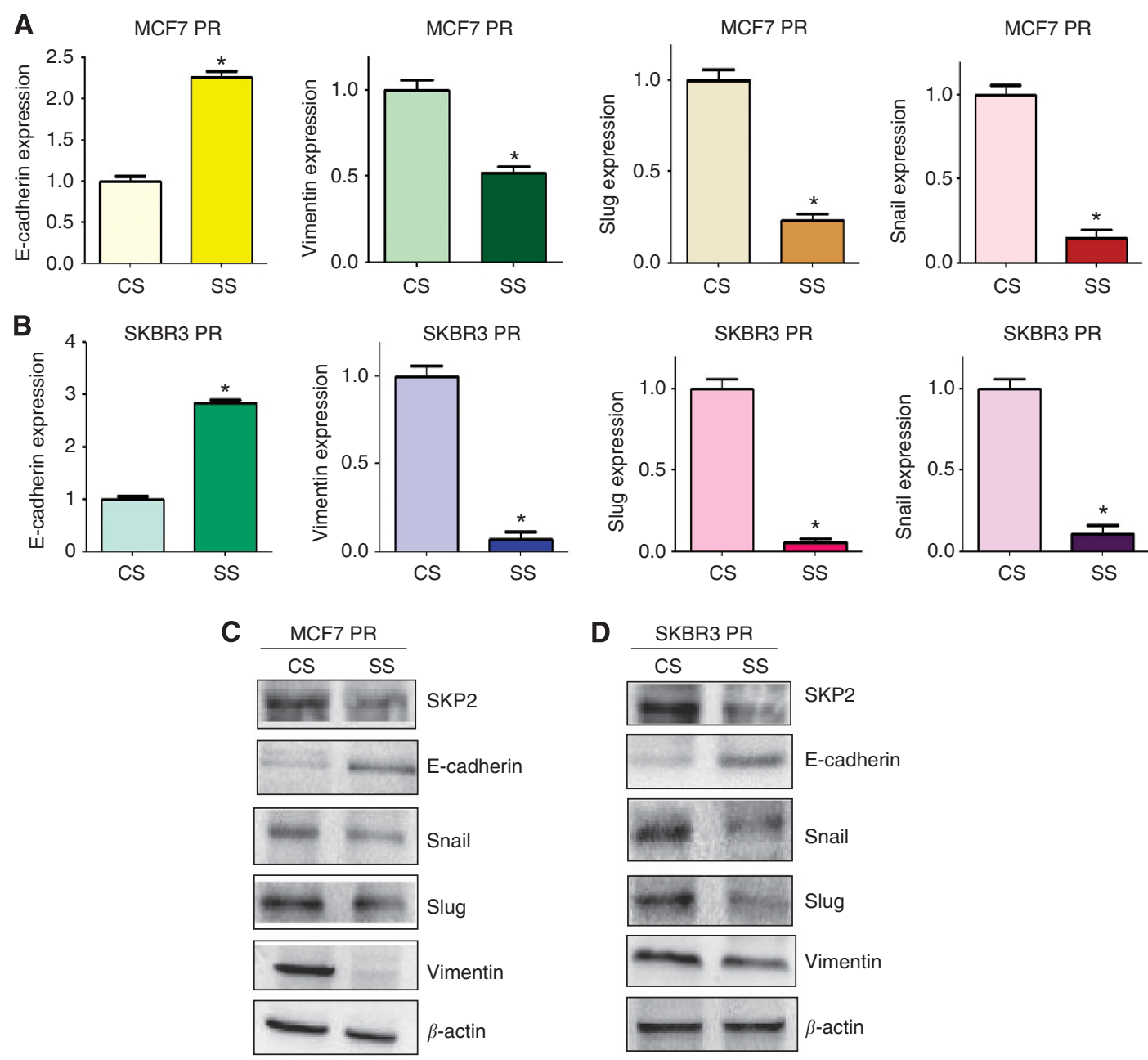

Figure 6. Depletion of Skp2 regulates expression of EMT markers in PR cells. (A and B) Real-time RT-PCR was performed to quantify mRNA expression of EMT markers in PR cells transfected with Skp2 siRNA. ${ }^{*} P<0.05$ compared with control siRNA. (C and D) Western blotting analysis was used to detect the expression of EMT markers in PR cells transfected with Skp2 siRNA. Abbreviations: CS $=$ control siRNA; SS $=$ Skp2 siRNA.

A
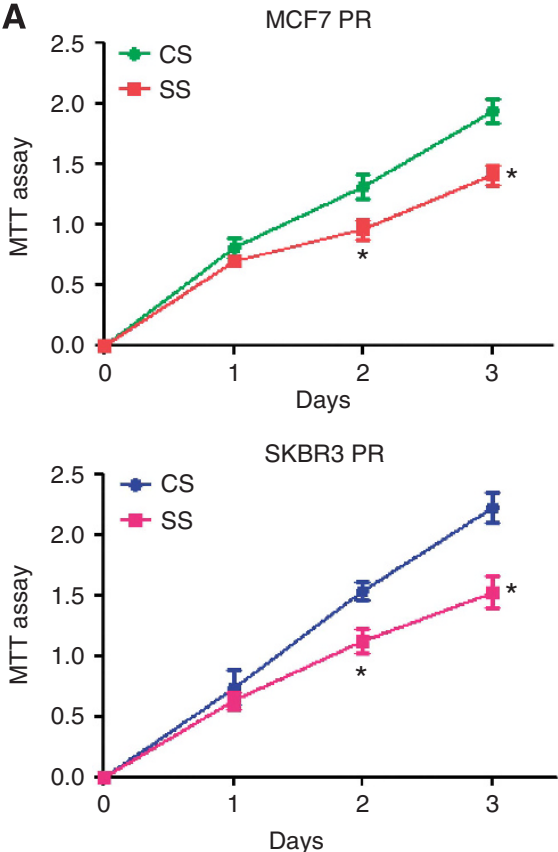

B

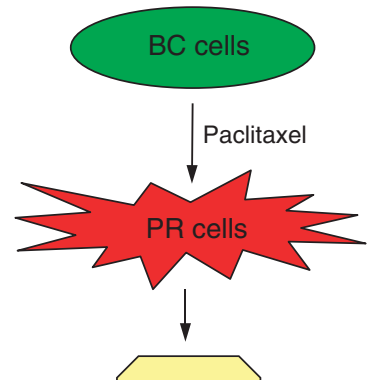

SKP2
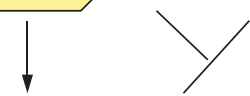

EMT

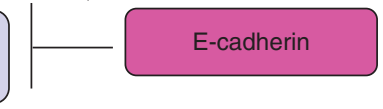

Migration

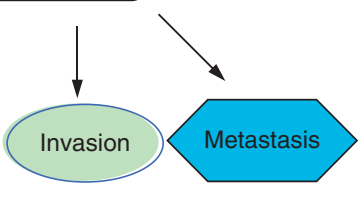

Figure 7. (A) MTT assay was performed in PR cells treated with Skp2 siRNA. ${ }^{*} P<0.05$ compared with control siRNA. (B) A proposed model for Skp2 in PR EMT-type cells. Abbreviations: BC= breast cancer; CS = control siRNA; EMT = epithelial-to-mesenchymal transition; $\mathrm{PR}=$ paclitaxel-resistant; $\mathrm{SS}=\mathrm{Skp} 2$ siRNA. 
these agents could be a novel and safer approach for the prevention of tumour progression and/or treatment of breast cancer.

\section{ACKNOWLEDGEMENTS}

The work was supported by grants from the National Natural Science Foundation of China (No. 81071848 and 81172087) and a project funded by the priority academic program development of Jiangsu higher education institutions, as well as a grant from a Key Program of Anhui Educational Committee (No. KJ2010A240 and KJ2013A184), and the Natural Science Foundation of Anhui (No. 1208085MH166).

\section{CONFLICT OF INTEREST}

The authors declare no conflict of interest.

\section{REFERENCES}

Ali S, Coombes RC (2002) Endocrine-responsive breast cancer and strategies for combating resistance. Nat Rev Cancer 2(2): 101-112.

Chan CH, Lee SW, Li CF, Wang J, Yang WL, Wu CY, Wu J, Nakayama KI, Kang HY, Huang HY, Hung MC, Pandolfi PP, Lin HK (2010) Deciphering the transcriptional complex critical for RhoA gene expression and cancer metastasis. Nat Cell Biol 12(5): 457-467.

Chan CH, Li CF, Yang WL, Gao Y, Lee SW, Feng Z, Huang HY, Tsai KK, Flores LG, Shao Y, Hazle JD, Yu D, Wei W, Sarbassov D, Hung MC, Nakayama KI, Lin HK (2012) The Skp2-SCF E3 ligase regulates Akt ubiquitination, glycolysis, herceptin sensitivity, and tumorigenesis. Cell 149(5): 1098-1111.

Chan CH, Morrow JK, Li CF, Gao Y, Jin G, Moten A, Stagg LJ, Ladbury JE, Cai Z, Xu D, Logothetis CJ, Hung MC, Zhang S, Lin HK (2013) Pharmacological inactivation of Skp2 SCF ubiquitin ligase restricts cancer stem cell traits and cancer progression. Cell 154(3): 556-568.

Chavez-MacGregor M, Gonzalez-Angulo AM (2013) Breast cancer in 2012: new drugs, new knowledge, new targets. Nat Rev Clin Oncol 10(2): 75-76.

Chen Q, Xie W, Kuhn DJ, Voorhees PM, Lopez-Girona A, Mendy D, Corral LG, Krenitsky VP, Xu W, Moutouh-de Parseval L, Webb DR, Mercurio F, Nakayama KI, Nakayama K, Orlowski RZ (2008) Targeting the p27 E3 ligase SCF(Skp2) results in p27- and Skp2-mediated cell-cycle arrest and activation of autophagy. Blood 111(9): 4690-4699.

Chen SY, Hu SS, Dong Q, Cai JX, Zhang WP, Sun JY, Wang TT, Xie J, He HR, Xing JF, Lu J, Dong YL (2013) Establishment of Paclitaxelresistant breast cancer cell line and nude mice models, and underlying multidrug resistance mechanisms in vitro and in vivo. Asian Pac J Cancer Prev 14(10): 6135-6140.

Davidovich S, Ben-Izhak O, Shapira M, Futerman B, Hershko DD (2008) Over-expression of Skp2 is associated with resistance to preoperative doxorubicin-based chemotherapy in primary breast cancer. Breast Cancer Res 10(4): R63.

De Craene B, Berx G (2013) Regulatory networks defining EMT during cancer initiation and progression. Nat Rev Cancer 13(2): 97-110.

Du F, Wu X, Liu Y, Wang T, Qi X, Mao Y, Jiang L, Zhu Y, Chen Y, Zhu R, Han X, Jin J, Ma X, Hua D (2013) Acquisition of paclitaxel resistance via PI3K dependent epithelial mesenchymal transition in A2780 human ovarian cancer cells. Oncol Rep 30(3): 1113-1118.

Einama T, Kagata Y, Tsuda H, Morita D, Ogata S, Ueda S, Takigawa T, Kawarabayashi N, Fukatsu K, Sugiura Y, Matsubara O, Hatsuse K (2006) High-level Skp2 expression in pancreatic ductal adenocarcinoma: correlation with the extent of lymph node metastasis, higher histological grade, and poorer patient outcome. Pancreas 32(4): 376-381.

Fujita T, Liu W, Doihara H, Date H, Wan Y (2008) Dissection of the APCCdh1-Skp2 cascade in breast cancer. Clin Cancer Res 14(7): 1966-1975.

Gheldof A, Berx G (2013) Cadherins and epithelial-to-mesenchymal transition. Prog Mol Biol Transl Sci 116: 317-336.
Hernandez-Aya LF, Gonzalez-Angulo AM (2013) Adjuvant systemic therapies in breast cancer. Surg Clin North Am 93(2): 473-491.

Holohan C, Van Schaeybroeck S, Longley DB, Johnston PG (2013) Cancer drug resistance: an evolving paradigm. Nat Rev Cancer 13(10): 714-726.

Huang H, Regan KM, Wang F, Wang D, Smith DI, van Deursen JM, Tindall DJ (2005) Skp2 inhibits FOXO1 in tumor suppression through ubiquitin-mediated degradation. Proc Natl Acad Sci USA 102(5): 1649-1654.

Huang HC, Lin CL, Lin JK (2011) 1,2,3,4,6-penta-O-galloyl-beta-D-glucose, quercetin, curcumin and lycopene induce cell-cycle arrest in MDA-MB231 and BT474 cells through downregulation of Skp2 protein. J Agric Food Chem 59(12): 6765-6775.

Huang HC, Way TD, Lin CL, Lin JK (2008) EGCG stabilizes p27kip1 in E2-stimulated MCF-7 cells through down-regulation of the Skp2 protein. Endocrinology 149(12): 5972-5983.

Inuzuka H, Gao D, Finley LW, Yang W, Wan L, Fukushima H, Chin YR, Zhai B, Shaik S, Lau AW, Wang Z, Gygi SP, Nakayama K, Teruya-Feldstein J, Toker A, Haigis MC, Pandolfi PP, Wei W (2012) Acetylation-dependent regulation of Skp2 function. Cell 150(1): 179-193.

Ishii T, Matsuse T, Masuda M, Teramoto S (2004) The effects of S-phase kinase-associated protein 2 (SKP2) on cell cycle status, viability, and chemoresistance in A549 lung adenocarcinoma cells. Exp Lung Res 30(8): 687-703.

Kajiyama H, Shibata K, Terauchi M, Yamashita M, Ino K, Nawa A, Kikkawa F (2007) Chemoresistance to paclitaxel induces epithelial-mesenchymal transition and enhances metastatic potential for epithelial ovarian carcinoma cells. Int J Oncol 31(2): 277-283.

Kamura T, Hara T, Kotoshiba S, Yada M, Ishida N, Imaki H, Hatakeyama S, Nakayama K, Nakayama KI (2003) Degradation of p57Kip2 mediated by SCFSkp2-dependent ubiquitylation. Proc Natl Acad Sci USA 100(18): 10231-10236.

Kong D, Wang Z, Sarkar SH, Li Y, Banerjee S, Saliganan A, Kim HR, Cher ML, Sarkar FH (2008) Platelet-derived growth factor-D overexpression contributes to epithelial-mesenchymal transition of PC3 prostate cancer cells. Stem Cells 26(6): 1425-1435.

Mallini P, Lennard T, Kirby J, Meeson A (2014) Epithelial-to-mesenchymal transition: what is the impact on breast cancer stem cells and drug resistance. Cancer Treat Rev 40(3): 341-348.

Qu X, Shen L, Zheng Y, Cui Y, Feng Z, Liu F, Liu J (2014) A signal transduction pathway from TGF-betal to SKP2 via Akt1 and c-Myc and its correlation with progression in human melanoma. J Invest Dermatol 1341): 159-167.

Radke S, Pirkmaier A, Germain D (2005) Differential expression of the F-box proteins Skp2 and Skp2B in breast cancer. Oncogene 24(21): 3448-3458.

Rico-Bautista E, Yang CC, Lu L, Roth GP, Wolf DA (2010) Chemical genetics approach to restoring p27Kip1 reveals novel compounds with antiproliferative activity in prostate cancer cells. BMC Biol 8: 153.

Roy S, Kaur M, Agarwal C, Tecklenburg M, Sclafani RA, Agarwal R (2007) p21 and p27 induction by silibinin is essential for its cell cycle arrest effect in prostate carcinoma cells. Mol Cancer Ther 6(10): 2696-2707.

Schuler S, Diersch S, Hamacher R, Schmid RM, Saur D, Schneider G (2011) SKP2 confers resistance of pancreatic cancer cells towards TRAIL-induced apoptosis. Int J Oncol 38(1): 219-225.

Siegel R, Naishadham D, Jemal A (2013) Cancer statistics, 2013. CA Cancer J Clin 63(1): 11-30.

Sonoda H, Inoue H, Ogawa K, Utsunomiya T, Masuda TA, Mori M (2006) Significance of skp2 expression in primary breast cancer. Clin Cancer Res 12(4): 1215-1220.

Tan Y, Qin S, Hou X, Qian X, Xia J, Li Y, Wang R, Chen C, Yang Q, Miele L, Wu Q, Wang Z (2014) Proteomic-based analysis for identification of proteins involved in 5-fluorouracil resistance in hepatocellular carcinoma. Curr Pharm Des 20(1): 81-87.

Thiery JP, Acloque H, Huang RY, Nieto MA (2009) Epithelial-mesenchymal transitions in development and disease. Cell 139(5): 871-890.

Tosco P, La Terra Maggiore GM, Forni P, Berrone S, Chiusa L, Garzino-Demo P (2011) Correlation between Skp2 expression and nodal metastasis in stage I and II oral squamous cell carcinomas. Oral Dis 17(1): $102-108$.

Tsvetkov LM, Yeh KH, Lee SJ, Sun H, Zhang H (1999) p27(Kip1) ubiquitination and degradation is regulated by the SCF(Skp2) complex through phosphorylated Thr187 in p27. Curr Biol 9(12): 661-664. 
Wang Z, Fukushima H, Inuzuka H, Wan L, Liu P, Gao D, Sarkar FH, Wei W (2012a) Skp2 is a promising therapeutic target in breast cancer. Front Oncol 1(57.

Wang Z, Inuzuka H, Zhong J, Liu P, Sarkar FH, Sun Y, Wei W (2012b) Identification of acetylation-dependent regulatory mechanisms that govern the oncogenic functions of Skp2. Oncotarget 3(11): 1294-1300.

Wang Z, Li Y, Ahmad A, Banerjee S, Azmi AS, Kong D, Sarkar FH (2011) Pancreatic cancer: understanding and overcoming chemoresistance. Nat Rev Gastroenterol Hepatol 8(1): 27-33.

Wang Z, Li Y, Kong D, Banerjee S, Ahmad A, Azmi AS, Ali S, Abbruzzese JL, Gallick GE, Sarkar FH (2009) Acquisition of epithelial-mesenchymal transition phenotype of gemcitabine-resistant pancreatic cancer cells is linked with activation of the notch signaling pathway. Cancer Res 69(6): 2400-2407.

Wu Q, Wang R, Yang Q, Hou X, Chen S, Hou Y, Chen C, Yang Y, Miele L, Sarkar FH, Chen Y, Wang Z (2013) Chemoresistance to gemcitabine in hepatoma cells induces epithelial-mesenchymal transition and involves activation of PDGF-D pathway. Oncotarget 4(11): 1999-2009.
Yang ES, Burnstein KL (2003) Vitamin D inhibits G1 to S progression in LNCaP prostate cancer cells through p27Kip1 stabilization and Cdk2 mislocalization to the cytoplasm. J Biol Chem 278(47): 46862-46868.

Yu ZK, Gervais JL, Zhang H (1998) Human CUL-1 associates with the SKP1/SKP2 complex and regulates p21(CIP1/WAF1) and cyclin D proteins. Proc Natl Acad Sci USA 95(19): 11324-11329.

Zardavas D, Baselga J, Piccart M (2013) Emerging targeted agents in metastatic breast cancer. Nat Rev Clin Oncol 10(4): 191-210.

Zheng WQ, Zheng JM, Ma R, Meng FF, Ni CR (2005) Relationship between levels of Skp2 and P27 in breast carcinomas and possible role of Skp2 as targeted therapy. Steroids 70(11): 770-774.

This work is published under the standard license to publish agreement. After 12 months the work will become freely available and the license terms will switch to a Creative Commons AttributionNonCommercial-Share Alike 3.0 Unported License. 